\title{
A Variable Precision Rough Set Model for Knowledge- assisted Management in Distance Education
}

\author{
https://doi.org/10.3991/ijet.v13i11.9602 \\ Wei Zhang \\ Wuhan University of Technology, Wuhan, China \\ Hubei Engineering University, Xiaogan, China \\ zwpc306@163.com
}

\begin{abstract}
To enable the teaching administrator to better obtain effective knowledge from a large amount of information to assist management and improve the efficiency and level of teaching management, a variable precision rough set model for knowledge assisted management of distance education was proposed. First, based on the theory of complete reduction and knowledge extraction, the proposed pedigree ambiguity tree was used as a strategy for obtaining complete reduction. An algorithm for obtaining a complete set of reductions was given. Then, by studying the process of knowledge extraction, a multiknowledge extraction framework was put forward. The process of data conversion was completely realized. Finally, experimental verification was performed. The results showed that the proposed model overcame the effect of noise data in real data and improved the efficiency of the algorithm. Therefore, the model has high universality.
\end{abstract}

Keywords - variable precision rough set, knowledge extraction, distance education, knowledge assisted management

\section{Introduction}

Distance open education is a new form of teaching in the world, and it is receiving increasing attention from the state and society. Open education has the characteristics of large scale of running schools, wide distribution of learners, and large differences among learners. For universities with remote open education, information-intensive management and knowledge-based management are its distinctive features. Especially in the era of knowledge economy, knowledge management has become the core competitiveness of the organization. Based on the large scale of education, managers of remote open education need to rely on various methods to obtain more and deeper knowledge to improve management efficiency and management quality. As a means of knowledge discovery, data mining technology can find hidden rules and knowledge from a large amount of data. Among them, rough set theory is one of the important methods of data mining.

The research content of attribute reduction in rough set theory has a natural connection with knowledge extraction. It is one of the effective tools for extracting knowledge 
from information systems. The information system is usually represented by a multirow (object) multi-column (attribute) _ dimension table, and knowledge extraction is done after attribute reduction in the information system. Attribute reduction does not change the classification of data in the information system. In other words, if there is a metric for the information, this metric will not change through attribute reduction. The knowledge extracted through attribute reduction is not only more concise and easy to understand, but also does not lose precision.

At present, the study of attribute reduction and knowledge extraction has received extensive attention from researchers at home and abroad. These studies have focused on extracting knowledge through minimal reduction. There are few references to theories, methods, and applications of complete reduction and knowledge extraction. In this context, the system needs to study the complete reduction and knowledge extraction.

\section{State of the art}

Domestic and foreign scholars have carried out a lot of theoretical research on the attribute reduction of variable rough set models. Yang et al. [1] considered that in the variable precision rough set model, the method of difference matrix is the theoretical basis of attribute reduction. However, this method is always computationally intensive. Therefore, to find a reduction faster, it needs to be effectively improved. The relative distinction of conditional attributes is defined. The smallest element in the difference matrix is characterized. This feature is used to create an algorithm that looks for all the smallest elements. Based on the algorithm for finding all the smallest elements, two algorithms were developed to find all reductions and one reduction in the variable precision rough set. Experiments show that the proposed method effectively reduces the amount of calculation. Zhao et al. [2] combined the variable precision fuzzy rough set with the interval type 2 fuzzy rough set to introduce the concept of variable precision interval type 2 fuzzy rough set (VPI2FRS). In addition, a new attribute reduction method has been developed within the VPI2FRS framework. Finally, the feasibility and effectiveness of the proposed reduction algorithm are verified by experiments. Abdullah and Jaddi [3] proposed a large flood algorithm for rough set attribute reduction. The "level" is nonlinear. In the attribute reduction process, the minimum information loss is used for reduction. Using a data set with discrete attribute values, the smallest subset of the original attribute set can be found in the rough set theory. The Great Flood algorithm optimizes this problem by using the lower bound "horizontal" to control the search space. Quantitative attribute reduction is more applicable and complex than qualitative reduction. Zhang and Miao [4] proposed an improved type of classification region and studied its preservation reduction. Then, the reduction target and preservation characteristics of the collection area are analyzed, and the reduction of the collection area is studied. Finally, the separability and rule consistency of the set region are verified, and a qualitative and quantitative double preservation reduction is established. Abdullah [5] proposed a new method of attribute reduction. The method is based on the record travel algorithm to solve the rough set attribute reduction problem. The 
algorithm has an isolated parameter, which is called deviation. It plays a key role in controlling the acceptance of a worse solution.

At present, the method of attribute reduction based on rough set and variable precision rough set model has been successfully used in different fields of research. Dai and $\mathrm{Xu}$ [6] proposed a fuzzy gain ratio attribute selection method based on fuzzy rough set theory, and applied this method to gene selection of tumor gene expression. It provides an alternative strategy for processing tumor data in gene expression or other applications. Liu et al. [7] proposed a new method for computing variable-precision rough set model reduction and applied to the evaluation of power production. Statistical evaluation is used to explain the economic significance of the attribute. Yang [8] uses spectral clustering method to discretize eigenvalues, so that rough set attribute reduction can be applied to feature selection. This method is applied to feature extraction and selection of ultrasound defect signals. It provides a technical reference for the classification of ultrasonic flaw detection signals.

In summary, the current research on variable precision rough set models is mostly theoretical. There is almost no distance education involved in the application. Aiming at the above research status, a variable precision rough set model for distance education knowledge-assisted management is proposed. It hopes to provide reference and help for knowledge management in distance education.

\section{Methodology}

\subsection{Reduction of complete attributes based on pedigree binary tree structure}

Obtaining all reductions is an NP-hard problem. In theory, the most straightforward strategy is to use enumeration to determine whether each combination of attributes is a reduction, thus obtaining all reductions. However, the main problem faced by this strategy is that as the number of attributes increases, the attribute combination increases exponentially by two, which is not suitable for data with a large number of attributes. Therefore, the researchers only go to partial reduction (multiple reduction). There are currently two mature strategies for achieving multiple reductions. In certain cases, it is also possible to obtain all reductions using these two strategies. The first is to obtain multiple reductions by non-nuclear attribute substitution. The core is to increase the number of reductions by replacing each other with non-nuclear attributes. This method is only possible to obtain a complete reduction set when the number of non-nuclear attributes of the seed reduction is consistent with the number of reductions. It lacks the replacement for non-nuclear attribute combinations, and the non-nuclear attribute replacement strategy uses a partially ordered search. The second is to use the method of group intelligence optimization to obtain multiple reductions. The core is to harness the intelligence of the group to find different reductions through random searches. The process is to randomly initialize the position of the individual in the population. By calculating the fitness function, the global optimal solution is found, and as a reduction output, multiple processes can be used to obtain multiple reductions. However, the group intelligence method requires a lot of parameters to be set, and different parameters have 
an impact on the reduction. The most important point is that the fitness function is often defined in terms of minimum reduction. If the length of any reduction of a complete reduction set is the same, it is possible to obtain a complete reduction set by using group intelligence. Neither the non-nuclear attribute substitution nor the group intelligence optimization strategy guarantees the completeness of the reduction set. Therefore, an orderly new strategy is needed to ensure the completeness of the reduced set. A complete attribute reduction based on pedigree binary tree structure is proposed.

A decision table is $T=(U, C, D, V, f)$. It is converted into a $\beta$ decision table $T_{\beta}=(U$, $\left.\mathrm{C}, \mathrm{D}, \mathrm{V}_{\beta}, \mathrm{f}\right)$. According to the decision-making monotonicity criterion, the decision value of $\mathrm{x}_{\mathrm{i}}$ is determined by formula (1):

$$
f\left(x_{i}, D\right)=\left\{\begin{array}{cc}
\arg \max _{D}\left\{\frac{\left|\left[x_{i}\right]_{C} \cap\left[x_{i}\right]_{D}\right|}{\left|\left[x_{i}\right]_{C}\right|}\right\} \text { if } \beta \leq \rho\left(x_{i}\right) \leq 1, \\
f\left(x_{i}, D\right) & \text { otherwises }
\end{array}\right.
$$

For $T_{\beta}$, its set of equivalence classes is $U / C=\left\{\left[x_{1}\right]_{C}, \ldots,\left[x_{i}\right]_{C}, \ldots,\left[x_{m}\right]_{C}\right\}$. Using the decision value formula (1), $T$ is converted into a distribution table $T_{D}=\left(U_{D}(\omega), C, D, V_{\beta}\right.$, f). According to the principle of the $\beta$ simplified decision table $T^{\prime}$, the number of objects in the distribution table is reduced. $\mathrm{U}_{\mathrm{D}}=\left\{\left(\mathrm{x}_{1}, \omega\left(\mathrm{x}_{1}\right)\right), \ldots,\left(\mathrm{x}_{\mathrm{i}}, \omega\left(\mathrm{x}_{\mathrm{i}}\right)\right), \ldots,\left(\mathrm{x}_{\mathrm{m}}, \omega\left(\mathrm{x}_{\mathrm{m}}\right)\right)\right\}$ is obtained. $\omega\left(\mathrm{x}_{\mathrm{i}}\right)$ is the distribution area label of the object $\mathrm{x}_{\mathrm{i}}$. The definition is as follows:

$$
\omega\left(x_{i}\right)=\left\{\begin{array}{l}
1 \text { if } \beta \leq \rho\left(x_{i}\right) \leq 1 \\
0 \quad \text { otherwise }
\end{array}\right.
$$

Only one object of the information system's equivalence class is stored in the distribution table. For the universe of variable precision rough set theory, especially for large data sets, it can greatly reduce the number of objects that need to be considered. The transformation of the decision table into the distribution table itself is a manifestation of narrowing the search space in the attribute reduction process according to the acceleration mechanism. Only one object in each equivalence class obtained by $\mathrm{U} / \mathrm{C}$ is retained in the distribution table. The number of objects in the distribution table is equal to the number of equivalence classes in which all objects are divided by all conditional attributes. Only one object in each equivalence class is retained in the distribution table. The decision value of the object is corrected according to the $\beta$ dominant decision value, and the distribution label $\omega(\mathrm{x})$ is added. The visible distribution table saves all the necessary information in the attribute reduction process while using the acceleration mechanism.

In the process of reduction, the selected attributes and the divided data are deleted, and the space for searching in the reduction can be further reduced, which is another application of the acceleration mechanism. After the selected attribute divides the data, the data that needs to be further divided is actually reduced, so the division ability of the remaining attributes will also change accordingly. Its ability to divide is not based on all sets of objects, but on data that is currently not available. As the combination of 
attributes increases, more and more data are divided. The decision table for further attribute reduction, the number of attributes and the number of objects are all decreasing. It should be noted that the undivided data is from the equivalence class of the selected attribute combination division, and the further attribute reduction process needs to utilize this equivalence class information. If the equivalence class information of the selected attribute is not retained, and only the reduced distribution table is used for attribute reduction, the necessary reduction information will be lost, which violates the basis of the acceleration mechanism.

Using the pedigree binary tree structure, attribute combinations are obtained to clearly classify different distribution state objects. A heuristic binary classification algorithm is designed. First, the data is binarized using a binary classification algorithm. A set of attribute combinations that can divide the target data from the non-target data is obtained. The definition of the reduction is used to make judgments. If this attribute combination is a reduction, it is placed in the reduction set. If it is not a combination of attributes, it is placed in the candidate reduction set. The attribute combination in the candidate reduction adds a new attribute through the next round of binary classification algorithm, and repeats the previous process until the candidate reduction set is empty, and the algorithm ends.

To obtain a complete reduction set, the complete attribute reduction algorithm (CVPR) based on variable precision rough set has four steps. The first step is to convert the decision table into a distribution table using equivalence class information. Compared with the decision table, the distribution table domain (number of objects) is significantly reduced, and the objects in the distribution table are added with information about the data distribution. In the second step, to obtain a set of attributes that satisfy the definition of the rough set reduction, the attribute order is defined and dynamically calculated. The third step generates a pedigree binary tree based on the attribute order. Finally, using the idea of binary classification, objects distributed in different classes in the decision table are gradually divided.

\subsection{Knowledge extraction based on complete attribute reduction}

Knowledge is a way for people to mine valuable parts by inductive, deductive, and comparative data. The knowledge system formed by it is used as prior knowledge, and this valuable information is transformed into knowledge. For the proposed complete reduction and knowledge extraction theory of variable precision rough sets, the multiknowledge extraction process mainly consists of the original data to the complete reduction set, and then from the complete reduction set to the multi-knowledge transformation process. The valuable part of the data is transformed into knowledge, which forms a multi-knowledge system. The data-to-knowledge transformation process is shown in Figure 1. 


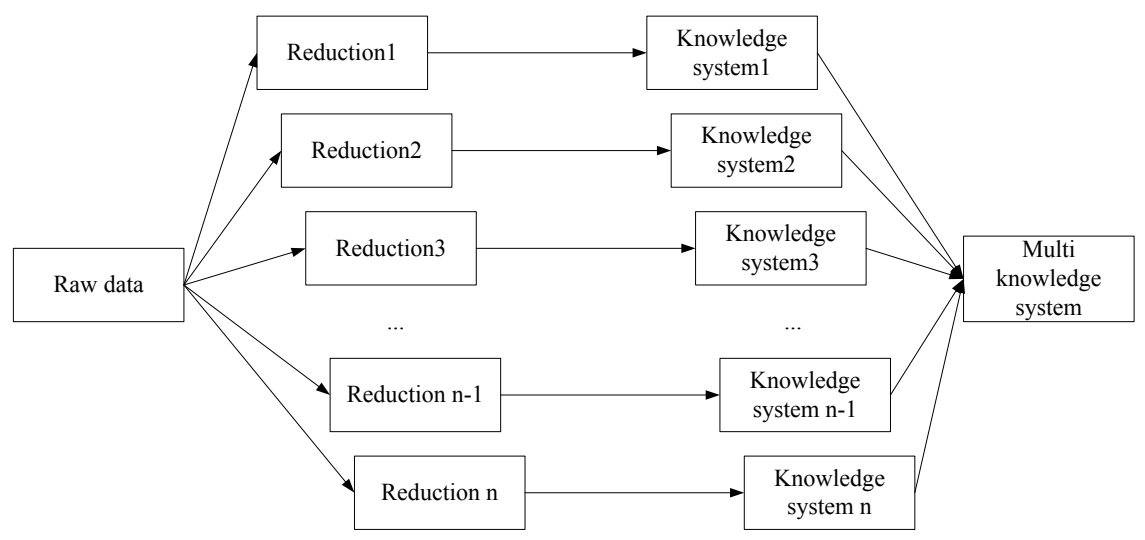

Fig. 1. The data-to-knowledge transformation process

In the past, attribute reduction algorithms generally obtained optimal or sub-optimal reductions, which only form a knowledge system. There is no process of knowledge system merging. The knowledge in the system comes from the same reduction. However, a complete reduction set will form multiple knowledge systems, and a multiknowledge system needs to be formed through the combination of knowledge systems. Compared with the knowledge system formed by single reduction, the relationship between reduction and knowledge in a multi-knowledge system obtained by complete reduction becomes complicated. Knowledge may come from one or some reductions. Therefore, after obtaining the multi-knowledge system, it is necessary to use the knowledge space structure to obtain the association information between the reduction and the knowledge.

According to the transformation process of existing data to knowledge, combined with the complete reduction and knowledge extraction theory of variable precision rough sets, a multi-knowledge extraction framework is constructed. The framework is mainly composed of data preprocessing, complete attribute reduction and multiknowledge extraction. Preprocessing is the foundation, reduction is the core, and knowledge is the performance. The collected data forms the original database. Then, a standard database is generated by the data preprocessing technique. A complete attribute reduction algorithm is used to obtain a complete reduction set in a standard database. A multi-knowledge system is formed by a complete reduction set. Finally, the multi-knowledge system is used as the background knowledge to obtain the two-layer structure of the knowledge space, and the association information between reduction and knowledge is obtained. Multi-knowledge extraction framework is shown in Figure 2 . 


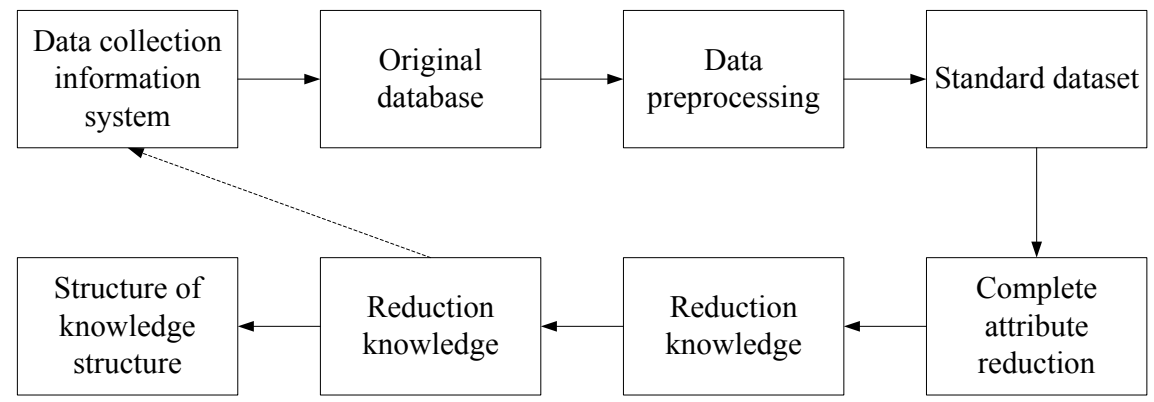

Fig. 2. Multi-knowledge extraction framework

Based on the previous knowledge extraction, the multi-knowledge extraction framework not only uses the complete attribute reduction algorithm to obtain a complete reduction set, but also forms a multi-knowledge system. Moreover, it is necessary to use the knowledge space structure to establish a complete reduction and multi-knowledge connection.

Data needs to be pre-processed before data is formed, and raw data is converted into standard data. The process of data preprocessing mainly includes cleanup, integration, transformation and specification. For the multi-knowledge extraction process, data preprocessing is embodied in missing value processing and continuous attribute discretization processing. The main operation for missing value processing is to delete and complete the data values. Deletion is the removal of data from missing values from the data set and is not used for further calculations. This method is useful if the sample size is large and the missing value data is small $(<5 \%)$. If there are many missing values, the missing values can be complemented by means such as mean or regression analysis. Rough sets are not suitable for directly processing continuous attributes. Therefore, before using the rough set for attribute reduction, continuous attributes in the information system need to be discretized. Discretization of continuous properties helps to reduce the effects of noise. In addition, it helps to simplify and improve knowledge in the process of multi-knowledge extraction and interpretation. Discretization methods are currently mainly supervised and unsupervised. Clustering is a supervised method, and the number of discretization needs to be set. The purpose is to make the data in the class have high similarity and the data in the same kind is not different. It considers the relationship between attributes and uses the distribution of data to obtain discontinuous points of continuous attributes, but the method of clustering is not conducive to processing data with uneven distribution. Information entropy selects discontinuities, which is an unsupervised way. It does not need to set the number of discretizations. The idea is to divide the continuous attribute values of consistent decisions into one interval. Therefore, its computational complexity depends on the type of decision value. It is more suitable for processing data with less decision value.

The decision table contains a lot of useful knowledge. The data is divided according to the rough set model, and the knowledge obtained by the reduction is derived from the equivalence class of the reduced partition. For reduction, the knowledge confidence in the positive regional equivalence class is high, and the number of objects in the 
equivalence class is greater. Knowledge support is also high. However, different reductions acquire knowledge from positive areas, and such knowledge may have conflicting knowledge. It is caused by the expansion of knowledge. From reduction to knowledge, attributes that are not in reduction can take any value. Then, the knowledge gained by different reductions can produce situations where the conditional attribute values are exactly the same but the decisions are different. Therefore, knowledge expansion may lead to the emergence of conflicting knowledge.

When determining the decision value of knowledge, Bayesian formula can be used for prediction. For a knowledge $\mathrm{C}^{\#}$, it contains a set of conditional attributes $\left\{\mathrm{c}_{1}^{\#}, \ldots, \mathrm{c}_{\mathrm{t}}^{\#}\right)$. The decision attribute value field of the decision table is $\left\{d_{1}, \ldots, d_{u}\right\}$. For $C^{\#}$, the decision value is most likely $d=\arg \max _{i=1}^{u}\left(d_{i} \mid C^{\#}\right) . \mathrm{P}(*)$ indicates the probability of a certain knowledge occurring.

$$
\begin{aligned}
& P\left(d_{i} \mid C^{\#}\right)=\frac{P\left(C^{\#} \mid d_{i}\right) P\left(d_{i}\right)}{P\left(C^{\#}\right)} \\
& P\left(C^{\#} \mid d_{i}\right)=\prod_{j=1}^{t} P\left(c_{j}^{\#} \mid d_{i}\right)
\end{aligned}
$$

From formula (3), the most probable decision of knowledge $\mathrm{C}^{\#}$ and its probability value are shown in formula (4):

$$
d=\underset{j=1}{\arg \max } \frac{P\left(d_{i}\right) \prod_{j=1}^{t} P\left(c_{j}^{\#} \mid d_{i}\right)}{\sum_{i=1}^{u} P\left(d_{i}\right) \prod_{j=1}^{t} P\left(c_{j}^{\#} \mid d_{i}\right)}
$$

Complete reduction set is $\left\{\mathrm{B}_{1}, \ldots, \mathrm{B}_{\mathrm{m}}\right\}$. For knowledge $\mathrm{C}^{\#}$ on the reduction $\mathrm{B}_{\mathrm{j}}$, all knowledge from the positive region is as in formula (5):

$$
W_{j}\left(d_{i}\right)=\left\{x \mid \forall x \in T_{D}\left(B_{j}\right), \omega(x)=1, f\left(x, C^{\#}\right) \rightarrow f\left(x, d_{i}\right)\right\}
$$

$T_{D}\left(B_{j}\right)$ represents the knowledge that $\mathrm{B}_{\mathrm{j}}$ obtained through $\mathrm{T}_{\mathrm{D}}$. Then, for determining the decision $\mathrm{d}_{\mathrm{i}}$ among the knowledge obtained through the complete reduction set, the weight value of the $\mathrm{w}_{\mathrm{i}}$ is as shown in the formula (6):

$$
w_{i}=\sum_{j=1}^{m}\left|W_{j}\left(d_{i}\right)\right|
$$


The structure of knowledge structure needs to obtain the connotation and extension of the node. In addition, the connection relationship of the two-layer structure of the knowledge space needs to be obtained. The lexicographical order is a method for effectively obtaining extensions. The general method does not consider the case where all objects have the same attribute value and how to generate the maximum extension of the upper neighbor. Therefore, based on the above analysis, an optimized order-based knowledge structure construction algorithm is presented. In the proposed knowledge structure construction algorithm, the extension and connotation of the nodes in the structure and the upper neighbor information of the construction structure are output. This method also outputs a two-layer connection between reduction and knowledge. To enhance the stability of multi-knowledge background construction, knowledge with higher confidence can be selected to form knowledge background to construct knowledge structure.

Knowledge background is set to $\mathrm{K}=(\mathrm{U}, \mathrm{A}, \mathrm{I})$. The theoretical approach to all concepts of knowledge background is as follows: After the attributes in A are arranged in a certain order, $\mathrm{U}$ is recorded and forms an initial epitaxial table. Then, according to the order of the attributes, $h(a), a \in A$ is intersected with all the extensions in the current epitaxy table. Each intersection is a new concept. If the new concept is not in the table, it is written into the epitaxial table.

Let $U=\left\{x_{1}, \ldots, x_{i}, \ldots, X_{n}\right)$, to facilitate illustration, objects in $U$ are represented in the form of foot markers, that is, $U=\{1, \ldots, i, \ldots, n\}$. Any subset of $U$ is arranged in order from small to large. $\mathrm{X}_{1}, \mathrm{X}_{2} \subseteq \mathrm{U}, \mathrm{X}_{1}$ dictionary is smaller than the $\mathrm{X}_{2}$ definition formula as follows:

$$
X_{1}<_{i} X_{2}=\left(i \in X_{2}-X_{1}\right) \wedge\left(X_{1} \cap\{1, \ldots, i-1\}=X_{2} \cap\{1, \ldots, i-1\}\right)
$$

According to the lexicographical order, the first unequal elements from $\mathrm{X}_{1}$ and $\mathrm{X}_{2}$ are large in $\mathrm{X}_{1}$. The minimum extension formula that is greater than $X \subseteq U$ according to the lexicographical order is as follows:

$$
\begin{aligned}
& X \oplus i=g(h((X \cap\{1, \ldots, i-1\}) \cup\{i\})) \\
& i=\arg \max _{i \in U-X}\left(X<_{i}(X \oplus i)\right)
\end{aligned}
$$

For a given set $X \subseteq U$, the minimum extension obtained in lexicographical order is $X \oplus i . \mathrm{i}$ is the largest element in $\mathrm{U}-\mathrm{X}$ that satisfies the condition. Therefore, the minimum extension of $\forall X \subseteq U$ in lexicographic order can be found using equation (8). The idea is to check the value of any element in $U-X$ in descending order of value. When it comes to $X<_{i}(X \oplus i)$ for the first time, $X \oplus i$ is the new extension.

In the actual knowledge background, the value of the attribute may not be binary. Multi-value backgrounds need to be converted to binary backgrounds when constructing knowledge structures. If the type of an attribute value is greater than 2 , this attribute 
needs to be converted from a multivalue to a binary value. The complete reduction and knowledge extraction theory and method of variable precision rough sets are applied. If there are too many reductions in a complete reduction set, the multi-knowledge system will be very large. Strong knowledge is screened using the confidence of knowledge and confidence intervals. Strong knowledge is used to construct a stable knowledge structure, and the Hasse diagram is used to visualize the presentation.

\section{$4 \quad$ Result analysis and discussion}

The complete attribute reduction algorithm is implemented in $\mathrm{C}$ language, and the configuration of the computer is as follows. The processor is Intel CoreT ${ }^{\mathrm{M}} \mathrm{i5}-3230 \mathrm{M}$ CPU@2.60GHz, and the memory is 4G. From the UC Irvine machine learning dataset warehouse, 30 samples of different object numbers and attribute numbers were selected for algorithm performance testing. 30 data sets such as heart, iris, and glass data sets are often used in classification tests. Some new data sets such as Bench, Fertility, and MAGIC Gamma Telescope were also selected for testing the algorithm. The average number of conditional attributes for these data sets is 12 . The maximum number of conditional attributes is 60 . The number of objects with 10 data sets exceeds 1000 . The maximum number of objects is 19,020 , and the average number of objects is 1980 .

The WADF algorithm (worst-attribute-drop-first) is a method that can achieve multiple reductions. Its strategy is the idea of non-nuclear attribute replacement. With a semi-ordered mechanism, seed reductions are changed to get more reductions. Taking the WADF algorithm of the non-nuclear attribute replacement strategy as an example, the attribute reduction is compared with the CVPR algorithm using the pedigree binary tree structure strategy.

Table 1. Comparison of the number of different algorithm reductions

\begin{tabular}{|l|c|c|c|}
\hline \multicolumn{1}{|c|}{ Data set } & WADF & ME & CVPR \\
\hline Credit Approval & 60 & 42 & 60 \\
\hline heart & 109 & 63 & 109 \\
\hline Iris & 4 & 4 & 4 \\
\hline Lenses & 0 & 0 & 0 \\
\hline Liver Disorders & 9 & 4 & 9759 \\
\hline Ionosphere & 32 & 26 & 846 \\
\hline Statlog & 16 & 11 & \\
\hline
\end{tabular}

For smaller data sets, such as credit approval, heart, iris, and liver disorders, both methods can extract all reductions. However, for some of the more aggressive data sets, such as Ionosphere and Statlog, only the CVPR algorithm using the pedigree binary tree structure strategy can achieve complete reduction. The ME (Multi-knowledge extraction) algorithm is also an algorithm that can obtain multiple reductions, and uses 7 data sets to compare the complete reduction of the three algorithms. Since the ME algorithm acquires multiple reductions only by attribute substitution, the number of 
reductions obtained compared to WADF or CKE is small. The comparison information is shown in Table 1.

Group intelligence is a strategy for optimizing algorithms, which speeds up the convergence of the algorithm. Moreover, its initial value is characterized by randomization, and multiple reductions can be obtained by running the algorithm multiple times. Fuzzy particle swarm optimization (FSRSR), fuzzy genetic algorithm attribute reduction (FGARS) and dynamic reduction (DR) are all algorithms that can obtain multiple reductions by initial value randomization. The index of the algorithm reduction quality is the number of attributes in the cylinder. The parameters in the algorithm are set according to the recommended values. The FSRSR algorithm has higher quality than the FGARS and DR reduction. Therefore, the pedigree binary tree structure strategy is compared with the group intelligence strategy. The CVPR algorithm and the FSRSR algorithm are compared.

The Glass data with 6 decision values is selected to compare the FSRSR algorithm representing the group intelligence algorithm with the proposed CVPR algorithm. Figure 3 is a multi-reduction process diagram of the FSRSR algorithm. The number of consecutive runs in the FSRSR algorithm is 108 (six times the number of reductions). The last reduction was obtained at the 20th run of the algorithm. A total of 11 reductions were obtained during the operation. The first reductions were run on the 1st, $2 \mathrm{nd}, 3 \mathrm{rd}$, 4th, 5th, 7th, 14th, 16th, 17th, 19th and 20th, respectively, and the other processes did not receive a new reduction. The Glass dataset actually has 18 reduction results. Seven reductions were not obtained. The reductions obtained are all minimal reductions, and the reductions not obtained are all non-minimum reductions. For other data, such as heart and Credit Approval, the reduction obtained using the FSRSR algorithm is not a minimum reduction or a second reduction. The process of FSRSR multi-reduction is shown in Figure 3.

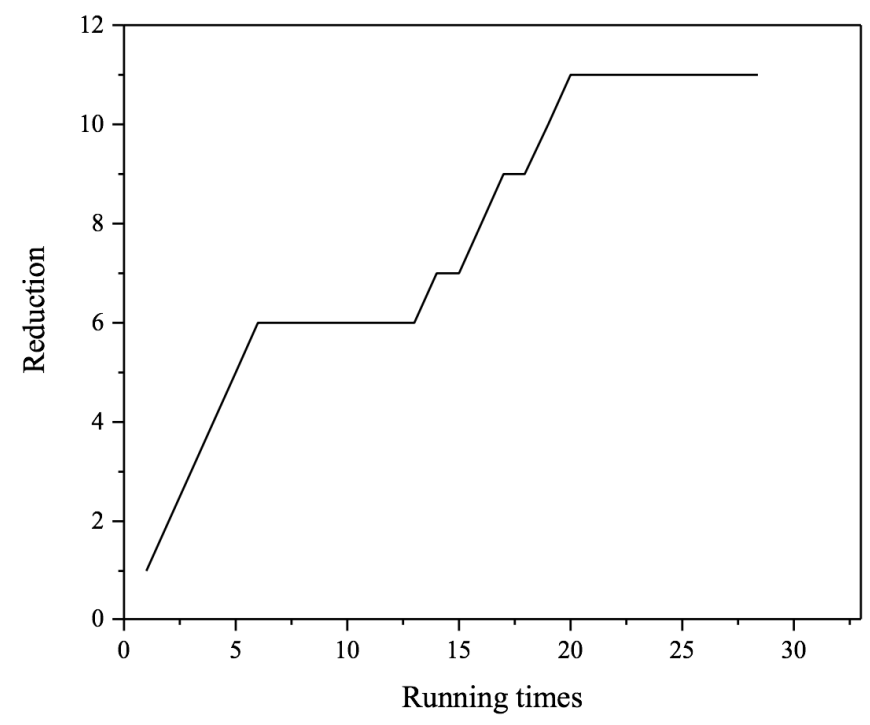

Fig. 3. The process of FSRSR multi-reduction 
Figure 4 is a time curve of the CVPR algorithm for the complete attribute reduction process of Glass data. The abscissa indicates the running time of the algorithm, and the ordinate indicates the reduction. Compared to the FSRSR algorithm, the CVPR algorithm does not require setting parameters. It gets all 18 reductions. The experiment fully demonstrates that the CVPR algorithm considering the distribution information and adopting the pruning strategy is a deterministic algorithm, and a complete reduction set can be obtained.

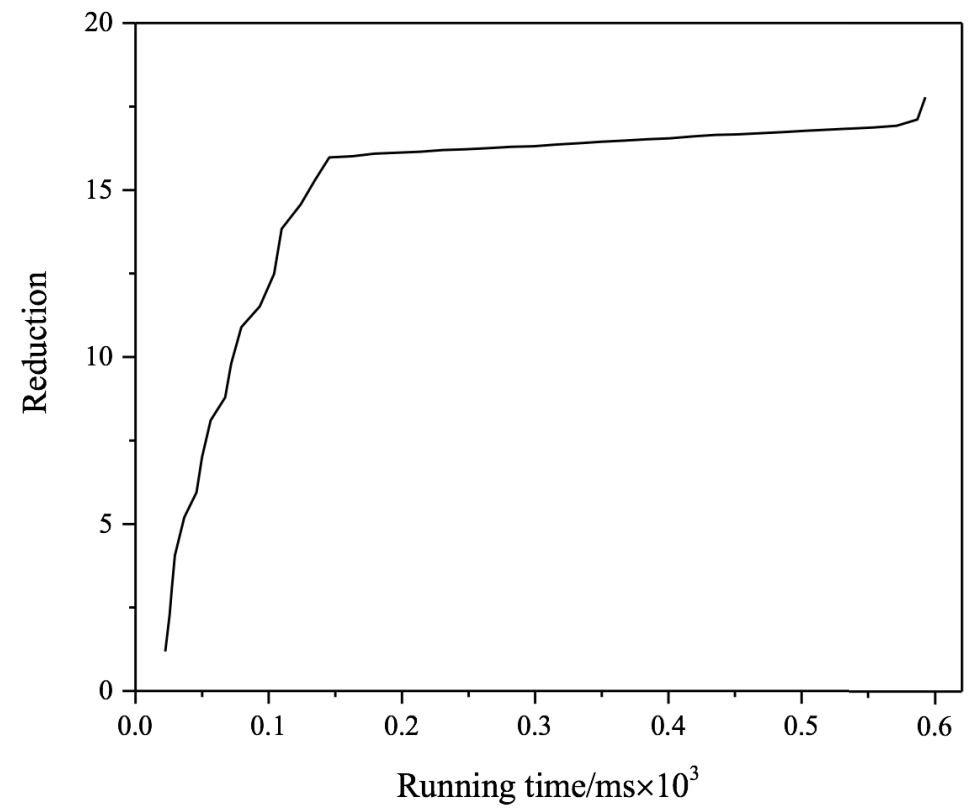

Fig. 4. Multi-reduction run time curve of Glass based on CVPR algorithm

For algorithms designed for non-nuclear attribute substitution and group intelligence optimization strategies, the attribute reduction algorithm is designed to achieve minimum reduction. The reduction of a large number of attributes is difficult to obtain. Multiple reductions can be obtained because the minimum reduction is often not unique. Therefore, a reduced set is basically a collection of minimum reductions. The completeness of the reduction cannot be guaranteed. The design of CVPR algorithm is based on complete attribute reduction and knowledge extraction theory. It is a deterministic search on the pedigree binary tree generated by the dynamic attribute order to obtain all reductions, which is obviously different from the strategy of using attribute substitution and group intelligence. The purpose of the CVPR algorithm using the pedigree binary tree structure is to ensure the completeness of the reduction. Moreover, the CVPR algorithm accelerates the convergence speed of its own algorithm by means of distribution table reduction, binary classification and pedigree tree pruning. 


\section{Conclusions}

By using the pedigree binary tree structure as the strategy to obtain a complete reduction set, a complete attribute reduction algorithm based on variable precision rough set is proposed. Then, by studying the knowledge extraction process, a multiknowledge extraction framework is proposed. It completely implements the process of transforming data into reductions to knowledge. Through the testing of standard database data, the proposed complete reduction algorithm can process relatively large-scale data. Compared with the existing multi-reduction algorithm, the complete reduction algorithm guarantees the completeness of the reduced set. The theoretical proof that the complete reduction algorithm obtains a complete reduction set is given. The proposed model has application value in distance education knowledge management.

\section{$6 \quad$ References}

[1] Yang, Y., Chen, D., Dong, Z. (2014). Novel algorithms of attribute reduction with var-iable precision rough set model: Neurocomputing, 139: 336-344 https://doi.org/10.1016/j.neucom.2014.02.023

[2] Zhao, T., Xiao, J., Ding, J., Chen, P. (2014). A variable precision interval type-2 fuzzy rough set model for attribute reduction: Journal of Intelligent \& Fuzzy Systems, 26(6): 2785-2797

[3] Abdullah, S., Jaddi, NS. (2013). Great Deluge Algorithm for Rough Set Attribute Re-duction: Journal of Information Science \& Engineering, 29(1): 49-62

[4] Zhang, X., Miao, D. (2014). Region-based quantitative and hierarchical attribute re-duction in the two-category decision theoretic rough set model: Knowledge-Based Systems, 71: 146161 https://doi.org/10.1016/j.knosys.2014.07.022

[5] Abdullah, MMS. (2015). A fuzzy record-to-record travel algorithm for solving rough set attribute reduction: International Journal of Systems Science, 46(3): 503-512 https://doi.org/10.1080/00207721.2013.791000

[6] Dai, J., Xu, Q. (2013). Attribute selection based on information gain ratio in fuzzy rough set theory with application to tumor classification: Applied Soft Computing Journal, 13(1): 211221 https://doi.org/10.1016/j.asoc.2012.07.029

[7] Liu, JNK., Hu, Y., He, Y. (2014). A set covering based approach to find the reduct of variable precision rough set: Information Sciences, 275(3): 83-100 https://doi.org/10.1016/ j.ins.2014.02.023

[8] Yang, P., Yang, Q. (2014). Empirical Mode Decomposition and Rough Set Attribute Reduction for Ultrasonic Flaw Signal Classification: International Journal of Compu-tational Intelligence Systems, 7(3): 481-492 https://doi.org/10.1080/18756891.2014.889877

\section{$7 \quad$ Author}

Wei Zhang is with Wuhan University of Technology, Wuhan, China and Hubei Engineering University, Xiaogan, China (zwpc306@163.com).

Article submitted 21 September 2018. Resubmitted 28 October 2018. Final acceptance 04 November 2018. Final version published as submitted by the author. 